drugs promoting the recruitment of opioid-producing cells. Second, the inhibition of selectin binding by fucoidin occurs without any change in the baseline pain sensitivity response. Fucoidin treatment only blocks analgesia induced by either CRF or the physiological response to systemic stress (in this case, the cold water swim test).

These findings are consistent with the induction of local anti-hyperalgesic effects by immune-cell release of $\beta$-endorphins. Although it is possible that in some cases the inflammatory and nociceptive response to injury is sufficient to promote a systemic stress response capable of causing opioid release, in other cases the immune cell-mediated analgesic activity may require the co-administration of agents such as CRF receptor agonists. Thus, analgesics that promote the adhesive interactions of opioid-positive immune cells alone may be inadequate. An essential, and as-yet undetermined, point is whether selectin-mediated immunocyte invasion relieves other types of pain be- sides that caused by inflammation.

Although there is an obvious need to address these and other issues, the observations of Machelska and co-workers provide exciting and provocative evidence for the existence of additional local pain regulatory mechanisms that integrate immune, opioid and nociceptor function. Such new information is sure to provide fertile ground for exploration of novel approaches to pain control, including the development of analgesics that target the immune system and lack the side effects of current pain killers.

1. Machelska, H., Cabot, P.J., Mousa, S.A., Zhang, Q. \& Stein, C. Pain control in inflammation governed by selectins. Nature Med. 4, 1425-1428 (1998).

2. Mendell, L.M. \& Wall, P.D. Responses of single dorsal cord cells to peripheral cutaneous unmyelinated fibers. Nature 206, 97-99 (1965).

3. Meyer, R.A., Campbell, J.N. \& Raja, S.N. Peripheral neural mechanisms of nociception. In Textbook of Pain (eds. Wall, P.D. \& Melzack, R.) pp. 13-44 (Churchill Livingstone, New York, 1994).

4. Liu, H., Mantyh, P.W. \& Basbaum, A.I. NMDA-receptor regulation of substance $P$ release from primary afferent nociceptors. Nature 386, 721-724 (1997).
5. Woolf, C.J., Mannion, R.J. \& Neumann, S. Null mutations lacking substance: Elucidating pain mechanisms by genetic pharmacology. Neuron 20, 1063-1066 (1998)

6. Heinricher, M.M. Cheng, Z.F. \& Fields, H.L. Evidence for two classes of nociceptive modulating neurons in the periaqueductal gray matter. J. Neurosci. 7, 271-788 (1987).

7. Yaksh, T.L \& Malmberg, A.B. Central pharmacology of nociceptive transmission. In Textbook of Pain (eds. Wall, P.D. \& Melzack, R.) pp. 165-200 (Churchill Livingstone, New York, 1994).

8. Meyer, R.A. \& Campbell, I.N. Myelinated nociceptive afferents account for the hyperalgesia that follows a burn to the hand. Science 213, 1527-1529 (1981).

9. Levine, J.D., Fields, H.L. \& Basbaum, A.I. Peptides and the primary afferent nociceptor. J. Neurosci. 13, 2273-2286 (1993).

10. Watkins, L.R, Maier, S.F. \& Goehler, L.E. Immune activation: The role of pro-inflammatory cytokines in inflammation, illness responses and pathological pain states. Pain 63, 289-302 (1995).

11. Levi-Montalcini, R., Skaper, S.D., Toso, R.D., Petrelli, L. \& Leon, A. Nerve growth factor: From neurotrophin to neurokine. Trends Neurosci. 19, 514-520 (1996)

Departments of Pharmacology and

Anesthesiology

University of Arizona Health Sciences Center

Tucson, Arizona 85724, USA

email: frankp@u.arizona.edu

\title{
Malaria: A 21st century solution for an ancient disease
}

\author{
The sequencing of the Plasmodium falciparum genome should reveal new parasite protein targets for drug \\ and vaccine development.
}

$D^{s}$ ESPITE MASSIVE EFFORTS to eradicate malaria in the 1950 s and early 1960s, today there are more humans infected with malaria than at any other time in history. More than 500 million people are infected with malaria worldwide, and one fourth of the world's population is at risk of infection. Furthermore, at least 2.5 million children die each year of malaria, most of them in Africa. Those children surviving chronic infection suffer a combination of anemia and immune suppression that leaves them vulnerable to other fatal illnesses. Drug resistance in the parasite is now widespread, further compromising prevention and treatment strategies. Malaria has confounded some of the best minds of this century. A hundred years after the discovery that mosquitoes transmit malaria, we still do not know enough about the disease to defeat it permanently ${ }^{1}$. However, a principal milestone in the field of malaria research has now been reached with the recent Science paper by Gardner et al. ${ }^{2}$ that presents the full-length sequence of one of the 14 chromosomes of Plasmodium falciparum, the organism that causes the most deadly form of human malaria. This se-

\section{DYANN WIRTH}

quencing achievement is the first for an organism as complex as $P$. falciparum and is particularly notable because it was feared that the intensely AT-rich Plasmodium genome would prove intractable to even the most sophisticated sequencing technology.

The Malaria Genome Sequencing Project is a cooperative effort between three sequencing centers-The Institute for Genomic Research (TIGR), the Sanger Center and the Stanford Genome Center-and the malaria research community with the goal of sequencing the entire genome of $P$. falciparum. It is sponsored by the National Institutes of Health, the Burroughs Wellcome Fund, the U.S. Department of Defense and the Wellcome Trust, and seeks to define every gene in the organism and, armed with this information, to identify new targets for drug and vaccine development. The sequence of chromosome 2, reported by the TIGR and Department of Defense groups ${ }^{2}$, is proof that it will be possible to achieve this goal.
The $P$. falciparum genome presents the sequencing 'gurus' with a number of technical hurdles to overcome. Most prominent of these is its remarkably high $\mathrm{A}+\mathrm{T}$ content (80.2 percent in chromosome 2). The sequencing of chromosome 2 required the investigators to adapt standard cloning techniques, modify computer programs that assemble the sequence, and develop new methods to finish the sequence analysis. The entire genome is approximately $30 \mathrm{Mb}$ in length and is arranged in 14 individual chromosomes that vary in length between wild-type isolates.

Chromosome 2 is just under $1 \mathrm{Mb}$ in length and contains 209 predicted open reading frames. By comparing gene sequences in the parasite with those of other organisms, the investigators were able to identify homologues for about 40 percent of the genes, with the remaining 60 percent falling into the class of unidentified open reading frames. This percentage of unidentified open reading frames is substantially higher than that reported in other organisms. The authors suggest that this may be due, in part, to the unusually high percentage of 


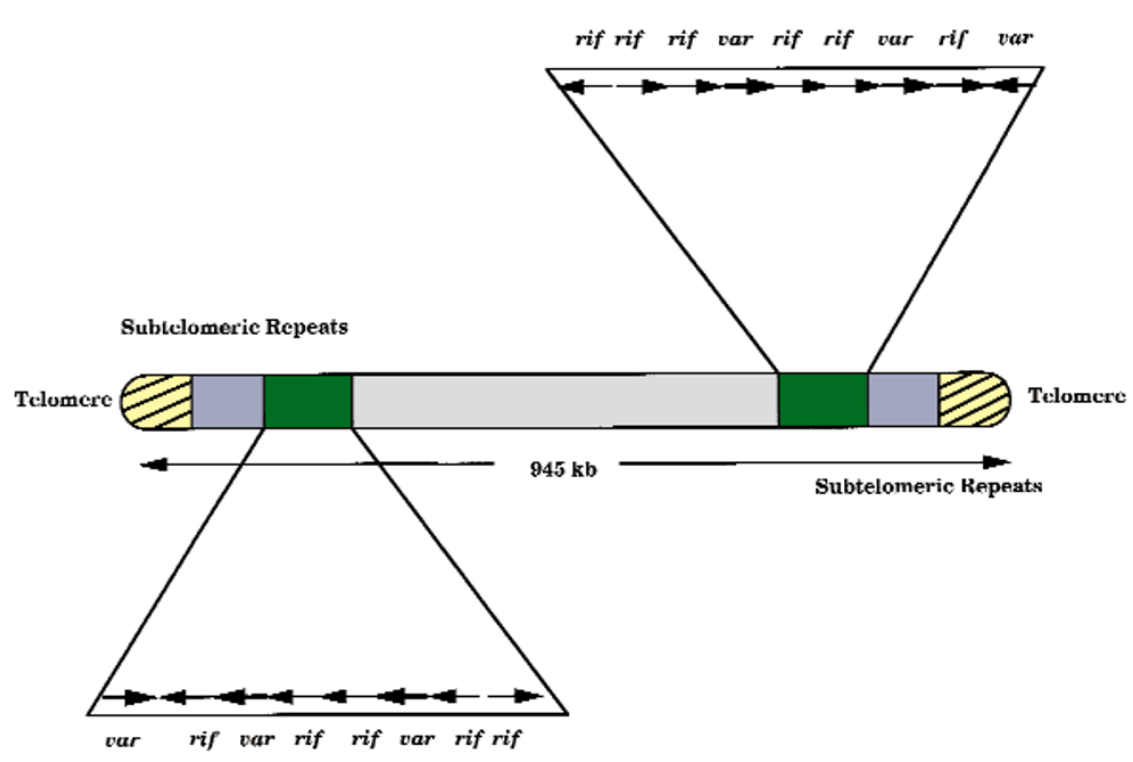

Chromosome 2 of Plasmodium falciparum. The availability of the complete sequence of chromosome 2 of $P$. falciparum, the parasite causing the fatal form of human malaria, should enable the identification of new targets for drug and vaccine development. The var and rifin genes, arranged in subtelomeric repeats at both ends of the chromosome, may be potential drug or vaccine targets, particularly because the var genes encode (and the rifin genes are thought to encode) parasite antigens that are expressed on the infected erythrocyte surface.

proteins with non-globular domains predicted from the open reading frames of $P$. falciparum chromosome 2. (The function and importance of these nonglobular protein domains is unknown, although the investigators suggest that they may provide a selective advantage to the parasite.) An additional contributory factor is certainly that the malaria parasite has an unusual and complex life cycle, existing in multiple forms in both the human host and mosquito vector. Host-parasite interactions and immune evasion strategies require unique genes and proteins.

One of the most interesting features of chromosome 2 is the arrangement of families of related genes (see Fig.). At each end of the chromosome, there are two families of genes: the var genes, which encode variant parasite antigens expressed on the surface of infected erythrocytes, and the rifin genes encoding a previously cloned gene family of unknown function named for the Rif- 1 repeat element ${ }^{3}$. The arrangement of the rifin genes interspersed with the variant surface antigen genes, coupled with the discovery that the rifin genes, have signal sequences, suggests that the products of these genes are expressed on the erythrocyte surface and may be associated with variant surface antigens. Neither the spatial relationship of these gene families nor the juxtaposition of the sig- nal sequences could have been determined using either expressed sequence tag approaches or standard mapping techniques.

One of the immediate applications of the Malaria Genome Sequencing Project is the identification of new potential targets for drug development, and the chromosome 2 sequence yields several new candidates. Of particular interest are enzymes present in the malaria parasite that are drug targets in other organisms and enzymes that are unique to the parasite and not found in the host. One example from chromosome 2 that seems promising is the 3-ketoacyl-ACP synthase III (FabH), an enzyme in the Type II fatty acid synthase system that had been thought to be restricted to bacteria and plant plastid systems only. Additional research will be needed to determine whether such putative targets are indeed essential for parasite survival and whether they are amenable to drug development. The sequence gives us direct access to the genes and the research can move directly to the focused effort of target evaluation. Further comparison of the $P$. falciparum genome with those of related organisms will provide leads to conserved genes of unknown function that may play an essential part in parasite survival.

Information from the genome project may also help scientists to develop new
DNA-based vaccines (see page 1351 of this issue). Recent work from Hoffman's group demonstrates that a DNA vaccine based on the gene encoding the sporozoite surface protein elicits an immune response in humans ${ }^{4}$. New genes encoding putative surface proteins, such as the Rif-1 gene family, have been identified on chromosome 2 and some of these may warrant further investigation as potential vaccine targets. Furthermore, as new protein domains important in cellcell interactions are identified through biological and immunological analysis, related genes can be readily sought in the sequence database. This will provide immediate information regarding variant and conserved regions that will be useful for vaccine design.

Sequencing and assembly of the remaining thirteen chromosomes of $P$. falciparum is underway, with about 40 percent of the 6,500 predicted genes at least partially sequenced. This now permits us to think about future applications that will be possible when the entire genome sequence becomes available. Of particular interest is the analysis of gene expression at the level of the entire organism through the use of DNA microarray $^{5,6}$ or Serial Analysis of Gene Expression strategies ${ }^{7}$. These approaches have proved extremely powerful in the analysis of Saccharomyces cerevisiae gene expression, and have led to the identification of large groups of genes that respond when yeast are grown under different conditions or in the presence of drugs $^{8}$. It seems reasonable to assume that a similar multigene response will occur in $P$. falciparum after exposure to new vectors, drugs or the host immune response. Previous work has focused on single genes; the availability of the tools provided by the $P$. falciparum Genome Sequencing Project will now allow us to take a more integrative approach, analyzing the interaction of several genes in response to a single stimulus. The ability to dissect these kinds of complex responses is likely to bring a whole new dimension to our understanding of the parasite.

Finally, availability of the chromosome 2 sequence will allow for comparison of different populations and isolates of the parasite and the determination of sequence polymorphisms and allelic diversity $^{9}$. Comparison of sequences from recent parasite isolates with the reference strain sequence may provide insight into mechanisms of pathogenicity and virulence. Comparison of gene expression 
profiles should also provide clues to those genes that are essential for growth in the human host but are not required for growth in in vitro culture.

Genomic research promises new insight into mechanisms by which the malaria parasite operates and might be defeated. The challenge for the malaria research community is to bring this knowledge to the treatment and prevention of this deadly disease. Every twelve seconds, a child dies of malaria and the hope that this project brings is that the new knowledge created will lead to a fundamental and lasting change in that statistic.

1. Wirth, D.F. \& Cattani, J. Winning the war against malaria. Tech. Rev. 100, 52-61 (1997).

2. Gardner, M.J. et al. Chromosome 2 sequence of the human malaria parasite Plasmodium falciparum. Science 282, 1126-1132 (1998)

3. Weber, J.L. Interspersed repetitive DNA from Plasmodium falciparum. Molec. Biochem. Parasitol. 29 117-124 (1988).

4. Wang, R. et al. Induction of antigen-specific cytotoxic $\mathrm{T}$ lymphocytes in humans by a malaria DNA vaccine. Science 282, 476-480 (1998).

5. DeRisi, J.L., Vishwanath, R.I. \& Brown, P.O. Exploring the metabolic and genetic control of gene expression on a genomic scale. Science 278, 680-686 (1997).

6. Wodicka, L., Dong, H., Mittmann, M., Ming-Hsiu, H.
\& Lockhart, D.J. Genome-wide expression monitoring in Saccharomyces cerevisiae. Nat. Biotechnol. 15, 1359-1367 (1997).

7. Velculescu, V.E. et al. Characterization of the yeast transcriptome. Cell 88, 243-251 (1997).

8. Marton, M.J. et al. Drug target validation and identification of secondary drug target effects using DNA microarrays. Nature Med. 4, 1293-1301 (1998).

9. Winzeler, E.A. et al. Direct allelic variation scanning of the yeast genome. Science 281, 1194-1197 (1998)

\section{Department of Immunology}

and Infectious Diseases

Harvard School of Public Health

Boston, Massachusetts 02115-6021, USA

email:dfwirth@hsph.harvard.edu

\section{SOD1 aggregates in ALS: Cause, correlate or consequence?}

\section{Increased expression of wild-type SOD1 in mice with ALS does not slow disease progression or diminish the pathological changes in motor neurons.}

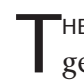
HE REPORT IN 1993 that mutations in the gene encoding $\mathrm{Cu} / \mathrm{Zn}$ superoxide dismutase (SOD1) cause some familial forms of the neurodegenerative disease amyotrophic lateral sclerosis (ALS) was greeted with the enthusiastic hope that this insight might lead to improved therapies for this lethal disorder ${ }^{1}$. Five years later, several mechanisms to explain the toxicity of mutant SOD1 protein have been postulated, but decisive experiments to distinguish which is correct are still awaited. Findings reported by Bruijn et al. ${ }^{2}$ in a recent issue of Science reinforce the view that neuronal toxicity is not the result of oxidative stress caused by the inability of mutant SOD1 to 'mop up' superoxide free radical. Their results suggest that mutant SOD1 may cause neurodegeneration by an alternative mechanism.

Among the explanations of how mutant SOD1 might contribute to neurodegeneration in ALS are those that invoke a disturbance in copper catalysis. For example, it has been argued that diminished metal binding by the mutant SOD1 protein might result in enhanced copper release and direct coppermediated neuronal toxicity. Alternatively, mutations that result in a widening of the SOD1 active site may allow promiscuous access of atypical substrates to SOD1-bound

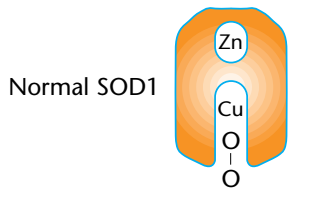

$$
\text { ROBERT H. BROWN, JR. }
$$

copper. Thus, if mutant SOD1 allows increased access of peroxynitrite (formed by the combination of superoxide anion and nitric oxide) to the active site, tyrosine nitration might follow ${ }^{3}$. Depending on the targets of this nitration (for example, neurofilaments or receptor tyrosine kinases), some of the resulting adducts could be neurotoxic. Alternatively, hydro-

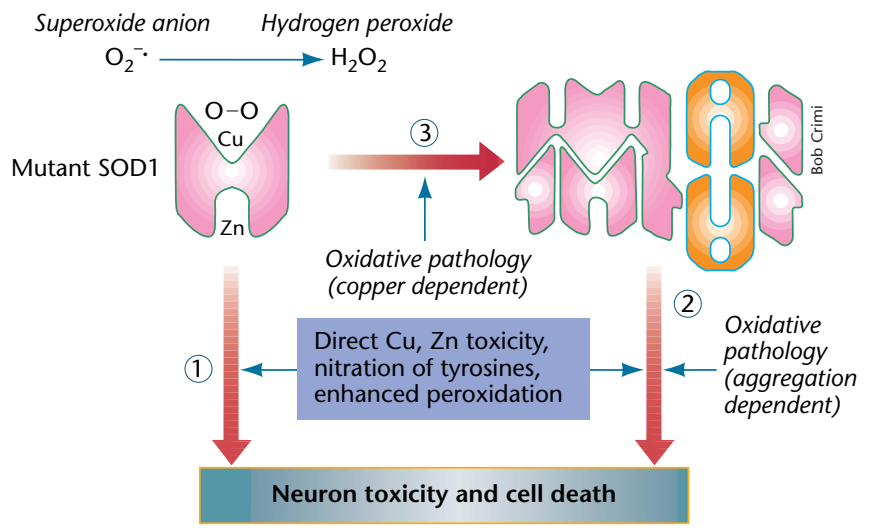

Mechanisms to explain the ways in which mutant SOD1 may be neurotoxic. The mutant SOD1 protein is assumed to be unstable with an active site that is relatively more accessible to substrates than the active site in the wild-type protein. The normal dismutation reaction (the breakdown of superoxide anion to hydrogen peroxide) mediated by wild-type SOD1, and that catalyzed by mutant SOD1 are shown (top). The mutant protein may be neurotoxic because of one or more catalytic reactions of copper (1) or through the formation of protein aggregates. The aggregates, in turn, may be a substrate for oxidative chemistry (2). It is possible that aggregate formation requires oxidative injury to mutant SOD1 (3). gen peroxide $\left(\mathrm{H}_{2} \mathrm{O}_{2}\right)$ - a product of the SOD1-mediated breakdown of superoxide anion-might be a substrate for the mutant protein, participating in peroxidation reactions that produce damaging hydroxyl radicals ${ }^{4}$.

An alternative set of mechanisms to explain the neurotoxicity of mutant SOD1 invokes aberrant protein-protein interactions. It has been proposed that the mutant protein binds and perturbs targets that are normally inaccessible or that it forms aggregates that are neurotoxic. It is known that neurons and astrocytes in ALS neural tissues contain aggregates that show immunoreactivity for SOD1 and ubiquitin (the protein that targets other proteins for proteolytic destruction). Such aggregates are seen both in familial ALS that results from mutations in SOD1 and in sporadic ALS of unknown etiology ${ }^{5}$.

The studies by Bruijn and colleagues $^{2}$ in transgenic mice with mutant SOD1, which develop a murine form of ALS, provide further insight into the pathology of this disease. The investigators genetically manipulated the levels of wild-type SOD1 in ALS mice expressing the mutant SOD1 protein. The levels of wildtype SOD1 varied from sixfold greater than normal to nil. The authors make two important observations. First, the timing of disease onset 